\title{
On the Field Below SM, Analysis and Predictions
}

\author{
Jacques Consiglio ${ }^{1}$ \\ ${ }^{1}$ Labastidette, France \\ Correspondence: Jacques Consiglio, 52, Chemin de Labarthe, F31600 Labastidette, France. Tel: 33-687-493-664. \\ E-mail: Jacques.Consiglio@gmail.com
}

Received: July 3, 2014 Accepted: July 28, 2014 Online Published: July 30, 2014

doi:10.5539/apr.v6n5p1 URL: http://dx.doi.org/10.5539/apr.v6n5p1

\begin{abstract}
We previously showed that the elementary particles mass spectrum is not random at all and probably originates in resonances of a single field below the known forces and fields. Here we first improve our demonstration leading to a single field; we show how resonances are compatible with the standard model symmetries and give coherent mass equation parameters. On this basis, we build a toy model of the field below which is consistent with those parameters and with the resonance numbers of quarks and massive bosons. It enables computing the $\mathrm{W}^{ \pm}, \mathrm{Z}^{0}$, and $\mathrm{H}^{0}$ boson pole masses with high precision and the widths also come naturally. The resulting $\mathrm{W}^{ \pm}$mass is in close agreement with experimental data but it disagrees with the SM prediction. An important consequence is that nothing new should appear in the next LHC run because geometrical constraints show that there is probably nothing else to find. Finally, the fundamental resonance agrees with the Dirac condition; it explains the other coefficients of the theory $\left(\alpha\right.$ and $\left.\alpha_{S}\right)$ and shows the nature of the field.
\end{abstract}

Keywords: mass spectrum, resonance width, standard model, forces unification

\section{Introduction}

The predictive power of the standard model (SM) of particle physics, together with the conceptual and mathematical abstractions at its basis, has made it hardly thinkable that nature could fundamentally be a lot simpler than its model and that another step of reductionism is possible. However there are still unsolved problems; one of those concerns the origin of the elementary particles mass spectrum (charged leptons, quarks and massive bosons). In a recent paper (Consiglio, 2014) we showed that it suggests the existence of a unique field below SM forces.

Our reasoning is based on a resonance equation that matches all known or estimated masses:

$$
m=\mu+X /(K D+1 / N P)^{3}
$$

Where all entities have a classical origin:

- $\quad X$ is the reverse of a density assumed universal (g. $\mathrm{L}^{3}$, where $\mathrm{L}$ is the natural unit for lengths).

- $\quad K, N$, and $P$ are small integral numbers (or a fraction in one case).

- $\quad D$ is a distance specific of the particle group (charged leptons, quarks, or massive bosons).

- $\quad \mu$ is a small self-energy assumed specific of the particle group (that we compute for leptons).

Alternately, $1 / N P$ and $D$ can be seen as ratios of distances and then $X$ becomes a mass.

In the standard theory this equation is an anomaly. Then we try to understand better its significance and the resonance numbers and parameters. The intention at this point is to understand the field below or kill the idea; we eventually get a number of unexpected results which confirm its existence and its natural simplicity. Hence this approach, while it looks at first as though it will be incoherent with the SM, is found when worked out to address its natural foundations.

In this paper, we first re-explain in details how equation (1) comes and what we should expect from it in terms of resonance types numbers - it gives direct relations with the SM symmetries. We repeat the resonances found for each group of particles and show that we empirically find a perfect match with those relations. It gives a second evidence of the existence of the field below. Then we repeat and augment our initial argument and we re-interpret, in a simpler manner, the system of equations leading to a single field "below SM forces". Based on this simpler but sounder analysis, we understand how the $D$ parameters work; they imply two and only two types of field constituents. On this basis we build a toy model of the field that fits and authorizes predictions. 
Finally, we find numerical evidences related to the nature of the field below. The charge ratio $1 / 2 \alpha$ appears in different manners and then the field is interpreted as magnetic currents. It is trivially coherent with the parameters $D$ as analyzed before, but also with the toy model of the field. It leads to a single fundamental type of current which is only distinguished by its orientation in space-time. We show that the fundamental resonance $X$ agrees with the Dirac condition; it also explains the $D s$ and definitely shows the nature of the field.

\section{Context}

\subsection{The Resonance Concept}

Equation (1) is based on a semi-classical model; we first split the particle in two pieces. We initially assume a pressure field that cages the particle charge(s) and self-energy $(\mu)$ in a reduced volume; $\mu$ is a self-energy and not intrinsically a mass, but caging it is like putting a photon in a mirrors box; the mass of the box increases and the photon still moves. The initial idea is similar, though not identical, to the Poincaré stress (Poincaré, 1906).

De Broglie's $E=h v$ strongly suggests some form of resonance and the standard theory heavily uses the concept. We assume all particles are harmonics of the same fundamental of resonance length 1 (a natural unit of length); in the one-dimensional case, the pressure is a simple force, and harmonic resonance implies some integral number $M$ such that we have:

$$
m=X M+\mu
$$

where $X$ is a natural constant. But the self-energy $\mu$ is massless and propagates; it implies a double resonance, the change in phase of the resonance is $2 \pi N$ over a length $l$ while $\mu$ is caged and must have a possibly different change in phase $2 \pi P$ over a length lesser than $1 / N$. Hence the actual resonance number is a product $N P$ and we get:

$$
m=X N P+\mu=X /(1 / N P)+\mu
$$

Using $1 / N P$ is natural as it is the resonance length. Caging a massless particle requires some sort of mirror, a repelling force that applies to the particle self-energy and to the pressure field as well; precisely to the first resonance wall and to the self-energy $\mu$ (and it should logically involve some charges). There must be a residual distance $d$ between the first resonance wall and the inner circulation $\mu$ at which the force applies. It gives:

$$
m=\mu+X /(d+1 / N P)
$$

In three dimensions, the system that we describe becomes spherical; the one-dimensional force becomes a pressure and it applies to a surface. Mutatis mutandis we get:

$$
m=\mu+X /(d+1 / N P)^{3}
$$

Essentially, this is $P V=k T$ with a single molecule since the quantity $V=(d+1 / N P)^{3}$ is seen as the volume of the cage. Now contrary to the one-dimensional problem, we have three degrees of freedom and the resonance paths $(N$ and $P$ ) can have different geometries coming from radial and circular paths. Overall, depending on how we mix paths, we find three and only three resonance schemes that should logically be identified to the three SM symmetries; they impose different constraints on $N$ and $P$ :

- A double radial resonance: It is compatible with $N=P$ and can trivially be identified with $\mathrm{U}(1)$ and also with the Poincaré stress.

- A double circular resonance: It is also compatible with $N=P$ and the forces at work in the resonance correspond to a symmetry group that inverts rotation axis; it must then be identified to $\mathrm{SU}(2)$.

- A mixed resonance implies $N \neq P$ with a geometrical constraint between $\pi, N$ and $P$ since we must have a phase lock between the two paths; squaring a circle implies approximate equalities like:

$$
N P \pi \approx \text { an integral number }
$$

The forces at work in the resonance should correspond to a group that combines $\mathrm{SU}(2)$ and $\mathrm{U}(1)$, and it must be identified to $\mathrm{SU}(3)$ - because it must include three instances of $\mathrm{U}(1)$, which are generated by the three inversions of $\mathrm{SU}(2)$, and similarly one or two instance of $\mathrm{SU}(2)$ generated by $\mathrm{U}(1)$, and this is a property of $\mathrm{SU}(3)$ which includes three instances of $\mathrm{U}(1)$ and two of $\mathrm{SU}(2)$.

Hence the resonance concept implies three symmetries that come close, if not identical, to the known ones.

The distance $d$ must also depend on $1 / N P$ because the mass (or energy) depends on the distance $(d+1 / N P)$ and it implies a potential - more exactly two potentials that add-up, one is $1 / N P$ and the other is $d$. Using quantum physics, a potential is quantized; $1 / N P$ is already quantized, and then $d$ is also quantized. The trick is to use $d=K D$ to evaluate this distance, with $K$ an integral number and $D$ a quantum of distance; it gives equation (1). 
The reasoning to this equation is simplistic and almost classical; although the $1 / N P$ and $K D$ can be seen as quantum concepts one should logically not expect much out of it, but as we shall see the true problem is that it works very well; let us now briefly re-explain the method used.

In order to match all known or estimated masses with (1), we analyze independently the three natural groups of particles (leptons, quarks and massive bosons) and we find the $N, P, K$, and $D$ separately for each group, with $D$ constant for a given group. Then each mass is computed from its ratio to the electron mass. We find numbers $N, P$, and $K$ which are small and show group-dependent regular patterns; they agree with the discussion here before: we find $N=P$ for leptons and bosons, and $N \neq P$ for quarks; the phase lock is trivial and comes naturally.

The next parameters to analyze are the $D s$, which are quite significant, because this is where forces apply, and then where they can be best understood. The standard model uses two important constants, namely $\alpha$ and $\alpha_{S}$ the coupling constants of QED and QCD respectively. They relate to the separation of leptons and quarks - the former at long range and the latter at very short range - and then directly to their masses. Then after matching (1) with existing masses we compare the $D s$ with $\alpha$ and $\alpha_{S}$; a consequence is that it appears natural and physical to express all $D$ s using only $\alpha$ and $\alpha_{S}$.

\subsection{Particles Resonances}

Leptons: Table 1 shows charged leptons resonances.

We find $K>0$, it denotes a repulsive force in agreement with the logic leading to equation (1) - as if a lepton charge repels the pressure field. We find $N=P$ for all particles, which it is not compatible with a mixed resonance scheme. It copes with a double radial resonance and then it corresponds to the Poincaré stress. It also copes with the electromagnetic field and its infinitely large spatial extension - the resonance does not need to be bounded.

Table 1. Electrons, muon, tau. $(*) \mathrm{MeV} / \mathrm{c}^{2}$

\begin{tabular}{llllll}
\hline Particle & $\mathrm{P}$ & $\mathrm{N}$ & $\mathrm{K}$ & Computed $\left(^{*}\right)$ & Measured $(*)$ \\
\hline Electron & 2 & 2 & 2 & 0.510998928 & $0.510998928(11)$ \\
Muon & 5 & 5 & 3 & 105.6583715 & $105.6583715(35)$ \\
Tau & 9 & 9 & 5 & 1776.84 & $1776.82(16)$ \\
\hline
\end{tabular}

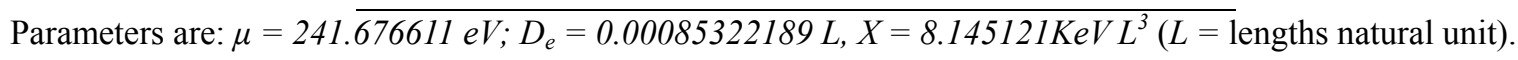

We define a priori a constant $\alpha_{S}$, (which we believe is closely related to the strong force coupling) from:

$$
\alpha_{S}=D_{e} / \alpha=0.1169221145
$$

To be compared with experimental values in the literature (e.g. $\alpha_{S}(M Z)=0.1181 \pm 0.0015$ ). We also find a numerical coincidence that can be interpreted as a fundamental property of the field or of the electron:

$$
4 \pi \alpha^{2}\left(m_{e}-\mu\right)=\sqrt{ } 2 \mu
$$

Hence $m_{e}$ and $\mu$ are linked solely by $\alpha^{2}$, and $4 \pi / \sqrt{ } 2$ looks like a natural coefficient. It enables computing $\mu$ in equation (1) using only the electron mass. Then assuming the universality of mass ratios, the free parameters of (1) are only $\alpha, \alpha_{S}$, and integral numbers. It was shown (Consiglio, 2014) that this coincidence is in close agreement with Bethe's results on the hydrogen Lamb shift.

Quarks: Table 2 shows the resonance numbers we found for quarks. $K<0$ (wrongly) suggests an attractive force.

For the top quark, we compare to the world average (The ATLAS, CDF, CMS and D0 Collaborations, 2013) and the recently published CMS measurement (The CMS Collaboration, 2014) which are of similar precision; in general, quarks masses are not known precisely, and then the important points are the general fit (the shape of the curve) and the difference between $D_{q}$ and $D_{e}$ that is needed to match the top quark mass. The reverse density $\mathrm{X}$ is unchanged and it suggests universality; $D_{q}$ gives a coincidence quite similar to (3):

$$
D_{q}=D_{e}(1+\alpha)
$$

The expression of $D_{q}$ used to compute Table 2 is (4), but the acceptable range $\left[D_{e}(1+0.8 \alpha)<D_{q}<D_{e}(1+1.4 \alpha)\right.$ ] is not negligible. With respect to (3), we could define $\alpha_{S}=D_{q} / \alpha=0.11775336$ and it might be more relevant. But we will stick to (3) as it will later give simpler expressions. (In both cases, it links $D_{q}$ and $D_{e}$ to $\alpha$ and $\alpha_{S}$.) 
Table 2. Quarks resonances. $\left({ }^{*}\right) \mathrm{MeV} / \mathrm{c}^{2}$, Top $=$ direct measurement $-\left({ }^{1}\right)$ world average $(2014)-\left({ }^{2}\right) \mathrm{CMS}(2014)$

\begin{tabular}{lllllll}
\hline Particle & Charge & $\mathrm{P}$ & $\mathrm{N}$ & $\mathrm{K}$ & Computed $(*)$ & Estimate $\left(^{*}\right)$ \\
\hline Up & $2 / 3$ & 3 & 2 & -6 & 1.93 & $1.7-3.1$ \\
Down & $1 / 3$ & 3 & $19 / 7$ & -6 & 5.00 & $4.1-5.7$ \\
Strange & $1 / 3$ & 3 & 7 & -6 & 106.4 & $80-130$ \\
Charm & $2 / 3$ & 3 & 14 & -6 & 1,255 & $1180-1340$ \\
Bottom & $1 / 3$ & 3 & 19 & -6 & 4,285 & $4130-4370$ \\
Top & $2 / 3$ & 3 & 38 & -6 & 172,380 & $173,340 \pm 270 \pm 710\left(^{1}\right)$ \\
& & & & & & $172,040 \pm 190 \pm 750\left(^{2}\right)$ \\
\hline
\end{tabular}

We get $N \neq P$ in Table 2; it is only compatible with a mixed resonance, which in turn implies a phase lock between the circular and the radial components; the radial and circular paths lengths must be multiples. We easily find the expected relations with $N P \pi$, but also the opposite with $N P / \pi$ :

$$
\begin{gathered}
7 \times 3 \pi \approx 65.97 \approx 66 / 1.0004025 \\
19 \times 3 \pi \approx 179.07 \approx 179 \times 1.0003954 \\
(19 / 7) \times 2 \times 3 \pi \approx 51 \times 1.0032 \\
2 \times 3 \pi \approx 18.85 \approx 19 / 1.008 \\
3 \times 7 \times 19 / \pi \approx 127.0056=127 \times 1.00004444
\end{gathered}
$$

Note that (19/7) is a special case. It fits with $2 \pi$ instead of $3 \pi$ and then all other coincidences should be multiplied by 2 . Finally, the integral numbers in Table 2 are natural solutions to the problem of mixed resonances.

Massive Bosons: The SM considers that the $\mathrm{W}^{ \pm}, \mathrm{Z}^{0}$ and $\mathrm{H}^{0}$ acquire their masses from a unique potential which has a single minimum; in our logic, it should correspond to the same resonance and coefficients, as in Table 3.

Table 3. Massive Bosons Resonances $(*) \mathrm{MeV} / \mathrm{c}^{2}$

\begin{tabular}{lllllll}
\hline Particle & $\mathrm{P}$ & $\mathrm{N}$ & $\mathrm{K}$ & $\mathrm{NP}$ & Computed $(*)$ & Measured $\left(^{*}\right)$ \\
\hline $\mathrm{W}^{ \pm}$ & 12 & 12 & -2 & 144 & $80,384.2$ & $80,385 \pm 15$ \\
$\mathrm{Z}^{0}$ & 12 & 12 & -7 & 144 & $91,187.67$ & $91,187.6 \pm 2.1$ \\
$\mathrm{H}^{0}$ & 12 & 12 & -19 & 144 & 126,355 & $125,500 \pm 400 \pm 400$
\end{tabular}

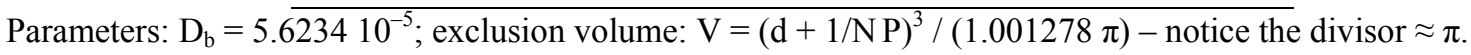

In the same logic as $(2-4)$, the distance $D_{b}$ can be approximated as follows:

$$
D_{b} \approx \alpha^{2}\left(1+\alpha_{S} / 2-\alpha_{S}^{2} / 6+\ldots\right)
$$

We find $K$ in $\{-2,-7,-19\}$ in Table 3, the same prime numbers as for quarks (Table 2,) but column $N$. It looks like an inversion of geometry with respect to quarks. The fact that $K D_{q}$ is constant in Table 2, while $1 / N P=$ 1/144 is constant in Table 3 also leads to the same idea.

We have $N=P$, and then the two resonances have the same orientation, but we find $K$ in $\{-2,-7,-19\}$ the same prime numbers as for quarks which resonance is mixed, and we need to divide the volume by $1.001278 \pi$ to compare with the electron mass (the factor was found empirically). Then we assume a double circular resonance and the length $K D_{b}$ is subtracted from an almost circular resonance path. Consequently, there is a physical constraint between the length $D_{b}$ and the circular path $\pi / N P=\pi / 144$.

Reasoning on geometry and using the fact that all $K s$ are prime numbers leads to the following coincidence:

$$
(1.001278)^{3} \pi / 144 \approx 2 \times 7 \times 19 D_{b}(\pi / 1.001278)^{1 / 3}
$$

This equation can be put in a more generic form (that we will later use) replacing 1.001278 by a variable $k$. It reads:

$$
k^{3} \pi / 144=266 D_{b}(\pi / k)^{1 / 3}
$$


Moreover, the two sides of (6.2) represent lengths, then taking their cube we get volumes.

It gives:

$$
\left(266 D_{b}\right)^{3}=k^{10} \pi^{2}(1 / 144)^{3}
$$

This equality compares, the volume of a cube of edge $266 D_{b}$, to that of a 4 -sphere $\left(V_{4}=\pi^{2} R^{4} / 2\right)$ divided by half its radius - with a correction $k^{10} \approx 1.013$ that is needed for squaring the circle (or rather to cubing the sphere). Firstly, it shows that $D_{b}$ is given and $k$ is a natural adjustment, because if nature was fine tuning $D_{b}$ we would find $k=1$; hence $D_{b}$ is not a natural constant, it is an interaction term, and it should be computed from theory. Secondly, it shows that our equations are not written the best possible way and then (6) is needed to get comparable quantities between electrons and bosons volumes; more importantly, it also means that the former depends on a radial quantity (for leptons, $1 / N P$ is then a radius and we have a double radial resonance) while the latter depends on a circular quantity (for bosons, $\pi / 144$ is a circumference and we have a double circular resonance). Then we can do better in order to identify this resonance with $\mathrm{SU}(2)$ using the numbers in Table 3.

Consider a circular resonance length equal to $2 \pi$, and based on $2 \times 7 \times 19=266$. The $K=-7$ and -19 in Table 3 imply two path lengths which are $L_{1}=2 \pi(1-7 / 266)$, and $L_{2}=2 \pi(1-19 / 266)$. The two resonances are in phase when $A L_{1}=B L_{2}$, with $A$ and $B$ integral numbers. We must solve the following phase equation:

$$
A \times 2 \pi(266-7) / 266=B \times 2 \pi(266-19) / 266
$$

The trivial solution is:

$$
A=266-19=247 ; B=266-7=259
$$

We get:

$$
B-A=12 ; A \times 2 \pi(266-7) / 266=B \times 2 \pi(266-19) / 266=240 \times 2 \pi+\pi
$$

The main resonance number, 12 , appears on the left hand side of (7.3); then the two paths show a phase coherence between the pressure field and the particles charges, but they must also propagate in the opposite direction and it gives $N=P$. Now $\pi$ comes on the right hand side and it confirms $\mathrm{SU}(2)$ but for particles of spin $1 / 2$. Hence the spin 1 bosons have two inner currents of identical directions in space, and since the $\mathrm{H}^{0}$ is spin 0 it should have four inner currents with two opposite directions in space. The $W^{ \pm}(K=-2)$ has the same geometry as it uses $N=P=12$.

We just showed that the resonance numbers in tables 1,2, and 3 agree with the SM Lagrangians, firstly in masses, but also (for the proven part) in symmetries. This, in turn, implies the existence of a field "below SM symmetries" that holds and propagates the resonances. A unique field from which known symmetries emerge and probably no other.

On intuitive grounds, the quarks resonance should be frequently broken, resulting in a conundrum of absorption and emission if compared to electrons - something we should probably expect from quarks as they are confined and locally exchange color charge while electrons are free and exchange no apparent charge. A large part of the hadrons mass should be caused by the resonance leak; hence quarks + SU(3) should be closest to the field below.

\section{The Field Below}

\subsection{Analysis of the D Coefficients}

Let us now show the existence of this field from a different perspective. Putting the remarkable points of the Tables 1,2 , and 3 together with the resonance schemes and the expressions of the $D s$ we get the following system:

$$
\begin{gathered}
\text { Leptons: Radial } \rightarrow N=P ; K>0 \rightarrow D_{e}=\alpha_{S} \alpha \\
\text { Quarks: Mixed } \rightarrow N \neq P ; K<0 \rightarrow D_{q}=\alpha_{S} \alpha(1+\alpha) \\
\text { Massive Bosons: Circular } \rightarrow N=P ; K<0 \rightarrow D_{b} \approx \alpha^{2}\left(1+\alpha_{S} / 2-\alpha_{S}{ }^{2} / 6\right)
\end{gathered}
$$

So it seems that we missed an important point:

A circular path systematically comes with $K<0$. Hence at the opposite of what we said in first analysis it does not imply an attractive force. Repulsion between the outer shell and the inner charges on a circular path will reduce the diameter of the path and give $K<0$ (as discussed for bosons) - then we find only repelling forces at work on the $K$.

Now as mentioned before, the $D s$ are very significant, because this is the interface between the inner circulation and the outer shell resonance; this is where they influence each other, and where the field can be best understood. We have two coefficients in the system, $\alpha_{S}$ and $\alpha$, which seem to combine quite simply.

Denoting $X$ the mass giving field, $C$ color charge, $E$ electricity and " $\times$ " interactions, classification and trivial identification gives Table 4 . 
Table 4. Classification and minimal interpretation of coefficients

\begin{tabular}{lllll}
\hline Origin & Coefficient & Interaction & Interpretation & Logic \\
\hline (8.1) $D_{e}$ & $\alpha \alpha_{S}$ & $X \times E$ & & Leptons charge \\
(8.2) $D_{q}$ & $\alpha \alpha_{S}$ & $X \times E$ & & Leptons $\rightarrow$ Quarks \\
(8.2) $D_{q}$ & $\alpha \times\left(\alpha \alpha_{S}\right)$ & $X \times C$ & $X \times C=X \times(X \times E)$ & Quarks charge \\
(8.3) $D_{b}$ & $\alpha^{2}$ & $X \times X$ & \\
(8.3) $D_{b}$ & $\alpha \times\left(\alpha \alpha_{S}\right) / 2$ & $X \times C$ & $X \times C=X \times(X \times E)$ & Quarks $\rightarrow$ Bosons \\
(8.3) $D_{b}$ & $-\left(\alpha \alpha_{S}\right) \times\left(\alpha \alpha_{S}\right) / 6$ & $(X \times E) \times(E \times X)$ & & Leptons $\rightarrow$ Bosons \\
\hline
\end{tabular}

It shows how the $D s$ work: Each individual interaction adds a piece of coefficient - like potentials adding or subtracting. Interpretational details of Table 4 are given hereafter.

Leptons (8.1); charge E.

$X \times E \rightarrow \alpha \alpha_{S}$ : There is only one interaction; it just gives us its coefficient.

Quarks (8.2); charges $E$ and $C$.

$X \times E \rightarrow \alpha \alpha_{S}$ : Same as electrons, and independent of the quark electric charge.

$X \times(X \times E) \rightarrow \alpha \times\left(\alpha \alpha_{S}\right)$ : This is the coefficient for color charge; it shows that color is not a specific distinct charge but that it has the same nature and quantum as $X$ (in agreement with our short discussion on broken resonances.)

Massive Bosons (8.3): charges $E$ and C.

The interaction is a little more complex then with quarks. We have the same coefficients for the $\mathrm{W}^{ \pm}$and the $\mathrm{Z}^{0}$. One is electrically neutral and not the other. Still, we find coefficients related to electricity and color charge, and then those bosons are made of two fractional electric charges and two color charges. Then it is:

$X \times X \rightarrow \alpha^{2}$ : The resonance involves two charges $X$, it gives a distance $\alpha^{2}$. This is the main resonance path and other coefficients will only augment or reduce it - they correspond to forces going thru this path.

$(X \times X) \times E \rightarrow \alpha \times\left(\alpha \alpha_{S}\right) / 2$ : The coefficient $\alpha^{2} \alpha_{S}$ comes with quarks color charge; it also shows that the inner circulation of a massive boson is equivalent to that of two quarks, and different of that of a lepton. The $1 / 2$ shows that in the $(X \times X)$, only one $X$ do interact with one $E$; unlike for quarks where the main resonance is not in $X \times X$.

$(X \times E) \times(E \times X)$ or $(X \times E \times X) \rightarrow-\left(\alpha \alpha_{S}\right) \times\left(\alpha \alpha_{S}\right) / 6$ : This coefficient corresponds to a retroaction of the main resonance on the electric charges; since $E$ is stuck between two $X$ it must have a direct relation with the particle stability or width.. We recognize the coefficient $\alpha \alpha_{S}$ from the leptons, but the $1 / 6$ is new.

At this point, we understand what is going on and we can deduce the missing coefficients in $D_{b}$.

$X \times X \times X \rightarrow-\alpha^{4}$ : Since $X \times X \rightarrow \alpha^{2}$ positive, and $K<0$, the force in $X \times X$ is attractive and then the charges involved in $X \times X$ are of opposite sign. Whatever the charge we put in between, the effect is the same, it reduces the main resonance and then the coefficient is negative -1 .

In a similar manner, each loop can be augmented with an additional $X$, and the operation repeated ad-infinitum; then the coefficients $-\alpha_{S}^{2} / 6$ must be multiplied by $\Sigma\left(-1^{n}\right) \alpha^{2 n}=1 /\left(1+\alpha^{2}\right)$ and $\alpha_{S} / 2$ by $\Sigma \alpha^{2 n}=1 /\left(1-\alpha^{2}\right)$.

The series make a small difference which is not negligible, and it gives:

$$
D_{b}=\alpha^{2}\left(1 /\left(1+\alpha^{2}\right)+\alpha_{S} / 2\left(1-\alpha^{2}\right)-\alpha_{S}^{2} / 6\left(1+\alpha^{2}\right)\right)
$$

Using $(9-6.2-1)$ we can compute the weak bosons masses with high precision but not the Higgs, because we just found that the $\mathrm{W}^{ \pm}$and the $\mathrm{Z}^{0}$ host the same charges as two quarks; this is fine for spin 1 particles, but maybe not for spin 0 ; although we guess four instead of two, it calls for a larger concept because we found evidences of distinct currents in the weak bosons resonances.

\subsection{One Field - A Minimal Picture}

Our goal is now to compute the Higgs boson mass, and then we need a model that is sufficiently close to reality, but we are in completely uncharted territory. We discuss unification from below (how to build elementary particles), while the SM sees distinct fields from above. We need to model at level 0 , not abstractions of level 1 or higher.

Our analysis of (8) imposes two types of field constituents, all massless, and all interacting with each other. Hence $E$ and $X$ should be the same thing, but they need to be distinguished by their orientation in space-time. 
The natural dissymmetry is time. Then assuming the symmetry of time is broken gives the following solution: Up-type quarks charges are currents going up the time, while down-type are currents going down the time and $X$ are the same thing but on the light cone. In this way, we have only one type of current; one symmetry that is broken in the time direction and also exists in the space directions. The idea is a universal quantum of current and originates in previous work (Consiglio, 2014).

The concept is coherent with the existence of $\mu$ and $X$.

Now since we have currents going up and down the time, we must assume that Cramer's interpretation of quantum mechanics (Cramer, 1986) is at least partly valid (it also comes in absorber theory); we will then use it as a guide where needed. From now, instead of charge we will use the term time-current.

We first represent currents; they have a sign ( + and - ) and a direction:

- Vertical arrows $\uparrow, \downarrow$ represent time-currents, up and down respectively (fractional electric charges);

- Oblique arrows $\lambda, K$ are space currents in $x, y, z$, a-priori on the light cone but causal and anti-causal.

We must now set a-priori rules for assembling currents.

They are:

- R1: Currents can assemble in all possible ways except for parallel direction and identical sign.

- R2: Space currents combine to form the interactors; time-currents are massive particles inner circulation.

- R3: Mass and forces emerge from the interaction of space and time-currents.

Implicitly, the apparent charge depends on the current direction. A negative electric charge is given by $\uparrow^{-}$for $-2 / 3$ or by $\downarrow_{+}$for $-1 / 3$. (Note the + and - as upper and lower indices, redundant with the arrow direction.)

And that is all. We have two types of currents (space or time) agreeing with the logic of inner and outer circulations, ( $\mu$ and $X$ respectively) fitting with an absorber scheme, and a single type of charge.

\subsection{Building Known Particles}

$\mathrm{SU}(3)$ seems to be the easy case because the triplet $\{\lambda+, \measuredangle-, \lambda-\measuredangle+\}$ fits with the arithmetic of color charge (the demonstration is trivial if one considers that $\lambda+\measuredangle+=\lambda-\measuredangle-=0$ ). Moreover the group made of the three time-currents $\left\{\uparrow^{+}, \downarrow_{-}, \uparrow^{-} \downarrow_{+}\right\}$fits with all known electric charges, and it is a broken form of the same triplet (in which the $\uparrow^{+} \downarrow_{+}$and $\uparrow^{-} \downarrow_{-}$do not exist) resulting from the dissymmetry of time.

But we found a difference between the inner circulation of $\mathrm{W}^{ \pm}$bosons and that of leptons; the latter does not come with the coefficients of color charge and then we assume that its currents merge $\uparrow^{-} \downarrow_{+} \rightarrow \uparrow^{-}$.

It gives:

- $\quad$ Up-type $\uparrow^{+}$, Down-type $\downarrow_{+}$, Electron-type $\left[\uparrow^{-} \downarrow_{+}\right] \rightarrow \mathfrak{1}^{-}$.

- Anti-Up-type $\uparrow^{-}$, Anti-Down-type $\downarrow_{-}$, Positron-type $\left[\uparrow^{+} \downarrow_{-}\right] \rightarrow \mathfrak{1}^{+}$.

Then U(1) massive particles correspond to $\mathfrak{\downarrow}^{-}$, and $\uparrow^{+}$and come from a broken SU(3).

Interestingly, the model is coherent with the fact that matter does not disintegrate alone. The up and down currents have the same charge and opposite direction - then for instance a proton cannot decay to a positron.

$\mathrm{SU}(2)$ is not so trivial, but there is actually no choice:

- A photon must be fully neutral and include a response wave hence $[\measuredangle-\measuredangle+\lambda-\lambda+]$;

- $\quad$ The $\mathrm{W}^{ \pm}$has the same charge as a lepton but it includes two time-currents; then $\left[\uparrow^{-} \downarrow_{+}\right]$or $\left[\uparrow^{+} \downarrow_{-}\right]$;

- The $Z^{0}$ includes two time-currents, it can be $\left[\uparrow^{+} \uparrow^{-}\right]$or $\left[\downarrow_{+} \downarrow_{-}\right]$or both.

The $\mathrm{H}^{0}$ should show a perfect symmetry, but nothing [-] does not fit with its $K=-19$; then it can only be $\left[\uparrow^{+} \downarrow_{+} \uparrow^{-} \downarrow_{-}\right]$. (Note that another neutral possibility would be $\left[\uparrow^{-} \uparrow^{+}\right]$; but this removes the color charge coefficient from (9) and gives a mass out of experimental range $\approx 121 \mathrm{GeV/c^{2 }}$.)

The neutrino and anti-neutrino can only be $[\lambda-\lambda+]$ and $\left[\boldsymbol{L - \measuredangle + ]}\right.$; two solutions like for the $Z^{0}$. Note that in this view, a neutrino has no response wave - it cannot be entangled and its quantum state has more degrees of freedom than, for instance, a photon.

And in theory this is all we need because nothing else is observed; however we must add $[\nearrow+\measuredangle+],[\nearrow-\swarrow-]$, and $\left[\uparrow^{+} \downarrow_{+}\right],\left[\uparrow^{-} \downarrow_{-}\right]$. Both give scissor effects; the former is seen as the de Broglie wave or the virtual photons field, the latter as a Cramer wave (as part of hadrons) - but none gives observable particles. 


\section{Leptons, Quarks, and Bosons Resonances}

One naturally wonders why we defined currents in this way. Firstly, they fit with our analysis of the coefficients $D$ if we systematically associate color charge to up and down-type currents (when not merged). Secondly, it seems to be the simplest possible design; it requires no extra dimensions and gives no new symmetry. Thirdly, we will show in this section that simple cross-relations exist between the currents as defined, the resonance numbers, and the electric charge of all known massive particles.

Let us first analyze the relation between quarks and massive bosons.

The prime numbers 2,7 , and 19 are central to the quarks and bosons resonances, and this must be linked to the composition of their inner circulations. But there is an inversion between quarks and bosons, for quarks these numbers multiply or divide $N$ while for bosons they give the $K$. Then we should search if our definition of currents is in relation with the $N$ multiplication factor - ideally, there is a one-to-one correlation.

The method consists in adding (resp. removing) the currents of a boson from a quark's; in parallel we multiply (resp. divide) the quark's $N$ by the boson's $K$, and then we check if we get the right charge and resonance.

For instance, the difference in $N$ between an up and a top quark is $N(u) \times K(H)=N(t)$; this should be equivalent to adding their currents: $\left[\uparrow^{+}\right]+\left[\downarrow_{+} \downarrow_{-} \uparrow^{+} \uparrow^{-}\right] \rightarrow\left[\uparrow^{+} \downarrow_{-} \uparrow^{-} \downarrow_{+} \uparrow^{+}\right]$. Then we assume the result is a top quark.

Let us continue with this idea starting with the up quark:

$$
\begin{array}{ll}
\text { - } & N(u) \times 19 \rightarrow N(t) . \text { Since }-19=K(H), \text { we add a } \mathrm{H}^{0}:\left[\uparrow^{+}\right]+\left[\downarrow_{+} \downarrow_{-} \uparrow^{+} \uparrow^{-}\right] \rightarrow\left[\uparrow^{+} \downarrow_{-} \uparrow^{-} \downarrow_{+} \uparrow^{+}\right] . \\
\text {- } & N(t) / 2 \rightarrow N(b) . \text { Since }-2=K(W), \text { we remove a } \mathrm{W}^{+}:\left[\uparrow^{+} \downarrow_{-} \uparrow^{-} \downarrow_{+} \uparrow^{+}\right]-\left[\uparrow^{+} \downarrow_{-}\right] \rightarrow\left[\uparrow^{-} \downarrow_{+} \uparrow^{+}\right] . \\
\text {- } & N(b) / 7 \rightarrow N(d) . \text { Since }-7=K(Z), \text { we remove a } Z^{0}:\left[\uparrow^{-} \downarrow_{+} \uparrow^{+}\right]-\left[\uparrow^{+} \uparrow^{-}\right] \rightarrow\left[\downarrow_{+}\right] .
\end{array}
$$

Note that the top quark breaks rule 1 as it has two parallel up-type currents. As we know it decays before it hadronizes, this unique property finds an echo here. Now, starting again with the up quark:

$$
\begin{array}{ll}
- & N(u) \times 7 \rightarrow N(c) \text {. Since }-7=K(Z), \text { we add a } Z^{0}:\left[\uparrow^{+}\right]+\left[\downarrow_{+} \downarrow_{-}\right] \rightarrow\left[\uparrow^{+} \downarrow_{-} \downarrow_{+}\right] . \\
\text {- } & N(c) / 2 \rightarrow N(s) \text {. Since }-2=K(W), \text { we remove a } \mathrm{W}^{+}:\left[\uparrow^{+} \downarrow_{-} \downarrow_{+}\right]-\left[\uparrow^{+} \downarrow_{-}\right] \rightarrow\left[\downarrow_{+}\right] .
\end{array}
$$

Although there is no corresponding boson we would like to add:

$$
\text { - } \quad N(s) \times 19 / 7 / 7 \rightarrow N(d) \text {. But } 19-7-7=-K(H)+K(Z)+K(Z) \text {, it gives: }\left[\downarrow_{+}\right] \rightarrow\left[\downarrow_{+}\right] .
$$

It actually corresponds to the full way back since we have: $19-7-7+2-2=-K(H)+K(Z)+K(Z)-K(W)+K(W)$.

Finally, we just have to multiply (or divide) the $N$ of a quark by the $K$ of a boson to get the right resonance, and to merge (or split) their currents to get the quark charge. But there is only one manner to make it, and it uses the dissymmetry of the two $Z^{0}$ definitions. We find, overall, some sort of broken resonance loop, as presented Figure 1 .

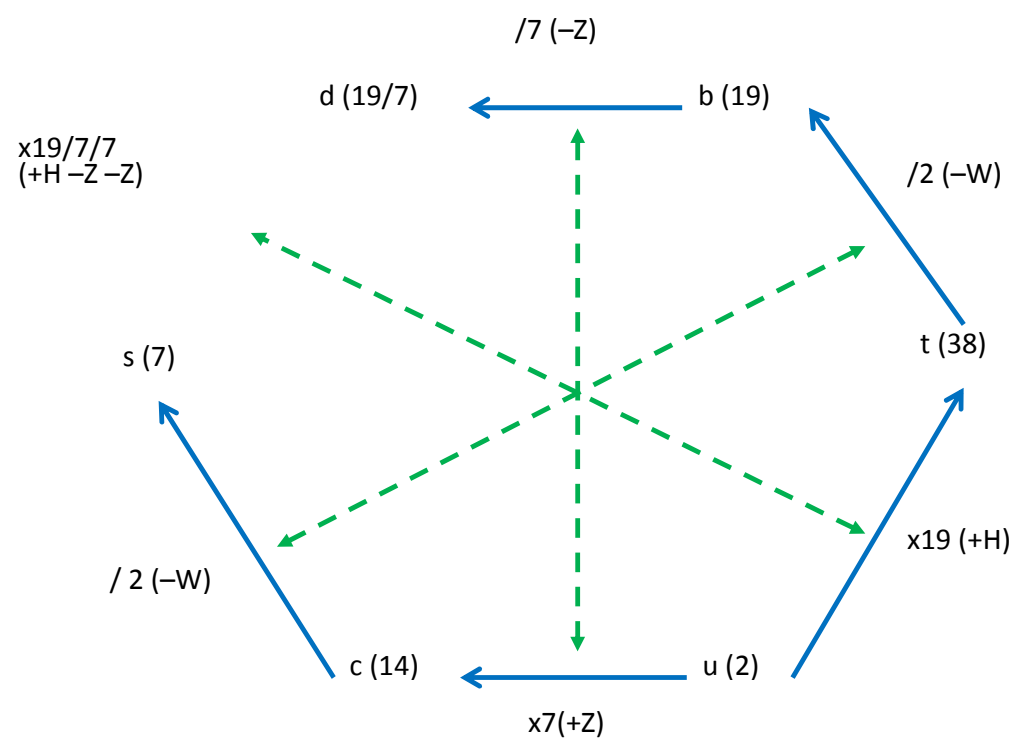

Figure 1. Quarks resonances: Going from one quark to the next by simple multiplication or division of its $N$ by one boson's $K$ (plain arrows). We find three distinct symmetries in resonance (dashed arrows) 
The symmetry of the loop is fully broken as we find three distinct types of transition. It opposes the $K s$ of the three massive bosons:

- $\quad \mathrm{W}^{ \pm}$: Two divisions by $2(b \rightarrow t$, and $c \rightarrow s)$.

- $\quad Z^{0}$ : One multiplication and one division by $7(d \rightarrow b$, and $u \rightarrow c$ respectively).

- $\quad \mathrm{H}^{0}$ : The ratios 19/7/7 and $19\left(s \rightarrow d\right.$, and $t \rightarrow u$ respectively); $\mathrm{H}^{0}-\mathrm{Z}^{0}-\mathrm{Z}^{0}-\mathrm{W}^{+}+\mathrm{W}^{+}$versus $\mathrm{H}^{0}$.

Presenting the circle (the arrows in Figure 1) in a different manner does not change the symmetries.

We also find that quarks of identical charge relate to sub-harmonics of a higher resonance, as shown Figure 2:

$$
\begin{array}{lll}
- & N(t) \times 7=N(c) \times 19=N(u) \times 19 \times 7=2 \times 19 \times 7=266 & (\rightarrow \text { charge }=2 / 3) \\
- & N(b) \times 7=N(s) \times 19=N(d) \times 7 \times 7=1 \times 19 \times 7=133 & (\rightarrow \text { charge }=1 / 3)
\end{array}
$$

Hence quarks can be seen as decayed state of a single high resonance, namely $N=266$, which must have the same origin as the $266 D_{b}$ in (6.2). Note that a quark with $N=266$ cannot exist, as well as $N=133$; because with those numbers (and using $P=3$, and $K=-6$, ) equation (1) gives a negative radius $\left(1 /(3 \times 133)-6 D_{q}<0\right)$.

It shows a perfect parallel for the second and third generations. But between the $s$ and the $d$ something special happens. As discussed previously, the $d$ resonance decays from the $b$, and not from the $s-$ on the other side, the $u$ resonance decays from the $t$ and from the $c$.

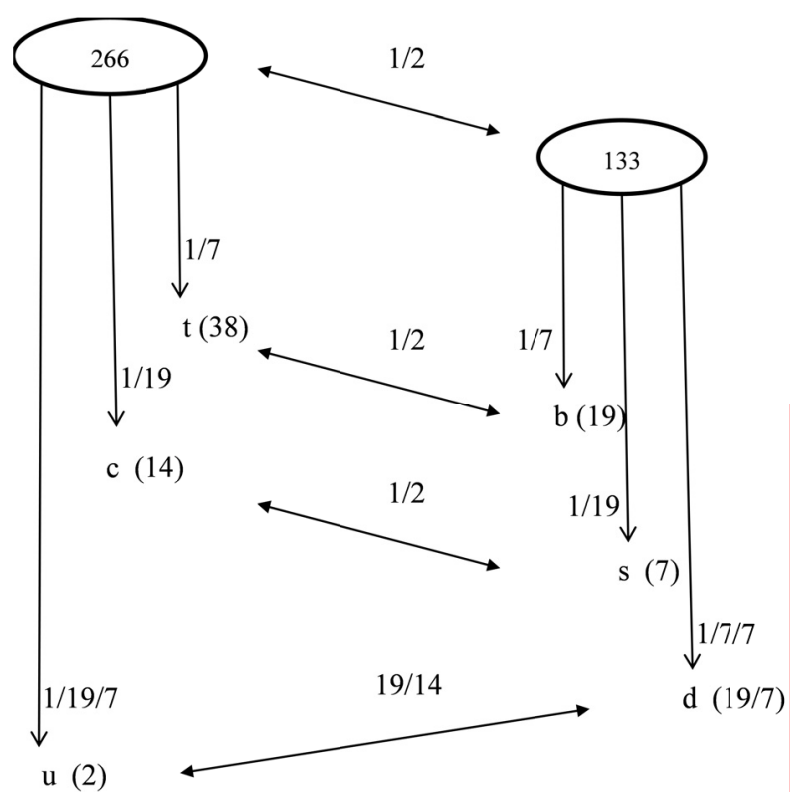

Figure 2. Quarks resonances as sub-harmonics of a single high resonance $(N=266)$

It gives a second manner to represent the relation between quarks and bosons currents and resonance numbers. Denoting (266) the high resonances, we find:

$$
\begin{array}{cc}
N(266) / K(H) / K(Z)=N(u) & N(266) / K(W) / K(Z) / K(Z)=N(d) \\
N(266) / K(H)=N(c) & N(266) / K(W) / K(H)=N(s) \\
N(266) / K(Z)=N(t) & N(266) / K(W) / K(Z)=N(b)
\end{array}
$$

where the "/" sign signifies simultaneously subtraction of currents and division of resonance numbers.

With respect to the CKM matrix (Table 5), we remark convergences with respect to the definition of currents: The highest coefficient is $V t b$, which corresponds to ratio 2 in $N ; N(t)=2 N(b)$. A perfect symmetry would require higher ratios for $V c s$ and $V u d$ and much smaller $V u s$ and $V c d$; but it is not so. Firstly the $d$ and $s$ quarks have the same time-currents definition; secondly we find a non-harmonic ratio between $N(u)$ and $N(d)$.

Hence the dissymmetry of the branching ratios in the KCM matrix is similar to that of the resonance numbers and of quarks time-currents. 
Table 5. The Cabbio-Kobayashi-Maskawa (CKM) matrix.

\begin{tabular}{lll}
$V u d=0.97427$ & $V u s=0.22534$ & $V u b=0.00351$ \\
$V c d=0.2252$ & $V c s=0.97344$ & $V c b=0.0412$ \\
$V t d=0.00867$ & $V t s=0.0404$ & $V t b=0.999146$ \\
\hline
\end{tabular}

Similarly, the mixed resonance of quarks also calls for a correspondence with leptons resonance numbers since both have a radial component.

A similar picture can be drawn for charged leptons, but now using an addition. (It seems, however, a little more far-fetched, but there is no way out; with three leptons we cannot find a scheme as rich and self-confirming its coherence as for quarks.) We guess:

$$
\begin{gathered}
\text { Electron: } N=P=2=-K(W)=N(u) ; K=2=-K(W) \\
\text { Muon: } N=P=5=-K(Z)+K(W)=N(s)-N(u) ; K=3=(-K(Z)-K(W)) / 3 \\
\text { Tau: } N=P=9=-K(Z)-K(W)=N(s)+N(u) ; K=5=-K(Z)+K(W)
\end{gathered}
$$

The addition of quarks $N$ seems natural to make a lepton; not the bosons $K$ but it is interesting to see the inversion between the right and left sides of $(10.2-10.3)$ : addition of bosons $K$ or quarks $N$ versus their subtraction.

Now assume that (10) has a geometrical origin; it implies a relation between $D_{e}$ and 266 like for $D_{b}$ in (6.2), and it should have a similar form since $D_{e}$ is radial and $\pi D_{b}$ is circular (remind that (6.2) links radial and circular paths).

We find:

$$
1 /\left(266 \pi^{1 / 3}\right)=D_{e} \times 3 \times 1.0028096
$$

It can be seen as a confirmation that (10) is physical, even with a discrepancy of $0.28 \%$, because once again we square a circle and the fit cannot be perfect. The factor 3 in (11) is not surprising, because we use the quarks $N$ which are directly related to 266 (and quarks use $P=3$ ). Then of course a fit similar to (11) exists for quarks with $D_{q}=D_{e}(1+\alpha)$ but with a discrepancy $\approx-0.45 \%$.

Let us then extend (10) to hypothetical leptons of $4^{\text {th }}$ and $5^{\text {th }}$ generations; they should use:

$$
\begin{gathered}
N=P=-K(H)+K(W)=N(b)-N(u)=17 ; K=(-K(H)-K(W)) / 3=7 \\
N=P=-K(H)-K(W)=N(b)+N(u)=21 ; K=-K(H)+K(W)=17
\end{gathered}
$$

The numbers we get look like a natural continuation of the series in Table 1; remarkably, all the $K s$ in (12) are prime numbers like for the three known generations. We also have for $N, P$ a pattern similar to the $2^{\text {nd }}$ and $3^{r d}$ lepton generations. But now it also requires that a relation exists between $D_{e}$, the leptons $K s$, including 7 and 17 , and $D_{b}$ and the bosons $K s$; but we do not know if our suite of bosons $K$ is complete. We only know that they use the same numbers as the quarks $N$; then using those which are prime with each other (hence including 19/7 even if the term "prime with" is abusive), we find a coincidence that closes the loop:

$$
D_{e}(19 \times 7 \times 2 \times 19 / 7) \approx 1.024 \times \pi D_{b}(2 \times 3 \times 5 \times 7 \times 17)
$$

The position of $\pi$ is appropriate since $\pi D_{b}$ is the true physical length which is part of the circular resonance path of bosons. It shows that $D_{e}$ and $\pi D_{b}$ are the divisors of a common length - but with an error of $2.4 \%$ which is large but can be explained: Leptons have merged currents while weak force bosons have two separated. Then the two quantities are not exactly comparable, and $2.4 \%$ is about half the difference between $D_{b}$ and $\alpha^{2}$.

In a manner similar to the quarks, the leptons charge is given by the currents of bosons used to compute its $N-$ using $(10-12)$. The $1 / 3$ in the $K$ is less obvious; it makes sense if we consider the number of parallel time-currents in the sum giving the lepton $K$.

- Electron: $\mathrm{A} \mathrm{W}^{ \pm}$has no parallel current, an electron has the same $K$.

- Muon: The sum giving $K$ is $Z^{0}+\mathrm{W}^{ \pm}$; it gives 3 parallel time-currents, sum their $K$ and divide by 3 , it gives 3 , which is the muon $K$.

- And so on with the tau and the hypothetical $4^{\text {th }}$ and $5^{\text {th }}$ generations.

Finally, our definition of currents has a close relation with the resonance numbers. It is also coherent with our analysis of the parameters $D$ as understood section 3, since it is in part the origin of the model. Moreover all the geometrical loops seem to be closed by $(7-11-13)$; we will then cautiously use this model to make predictions. 


\section{Predictions}

\subsection{Weak Force and Higgs Bosons Masses}

In Equation (9), only the coefficient $\alpha^{2} \alpha_{S} / 2$ and $-\alpha^{2} \alpha_{S}^{2} / 6$ come from the time-currents; the former is related to color-neutrality and is valid for any even number of time-currents, but the latter is not valid for the $\mathrm{H}^{0}$. The signs of weak bosons time-currents are opposites, and it gives the same coefficient $-1 / 6$. It means that the coefficient depends only on the number of possible mutual retroactions and that it is independent of the sign and direction of the current. For the 4 currents of the $\mathrm{H}^{0}$, we have 6 ways of feedback; the coefficient is then -1 in this case.Then, using (9), and updating the coefficient $-1 / 6$ to -1 for the Higgs, we find:

$$
\begin{gathered}
W^{ \pm} \text {and } Z^{0} \rightarrow D_{W Z}=\alpha^{2}\left(1 /\left(1+\alpha^{2}\right)+\alpha_{S} / 2\left(1-\alpha^{2}\right)-\alpha_{S}^{2} / 6\left(1+\alpha^{2}\right)\right)=5.6240490410^{-5} \\
H^{0} \rightarrow D_{H}=\alpha^{2}\left(1 /\left(1+\alpha^{2}\right)+\alpha_{S} / 2\left(1-\alpha^{2}\right)-\alpha_{S}^{2} /\left(1+\alpha^{2}\right)\right)=5.5633866410^{-5}
\end{gathered}
$$

Now we must use (6.2) to close the resonance paths, and we get the factors needed to compute the bosons masses:

$$
\begin{aligned}
& k_{W Z}=1.00128565 \\
& k_{H}=0.998033312
\end{aligned}
$$

Importantly there is no degree of freedom in the constants used or in the choice of integral numbers (even the $1 / 144$ is constrained by equation (6), and also from the fact that $12=19-7$ ); this is why the masses predicted using (14 -15 ) are of interest.

They are shown in Table 6 together with the SM prediction where relevant.

Table 6 Predicted Bosons Masses $(*) \mathrm{MeV} / \mathrm{c}^{2}$

\begin{tabular}{llllllll}
\hline Particle & $\mathrm{P}$ & $\mathrm{N}$ & $\mathrm{K}$ & $\mathrm{NP}$ & Computed $(*)$ & Measured $(*)$ & SM Prediction $\left(^{*}\right)$ \\
\hline $\mathrm{W}^{ \pm}$ & 12 & 12 & -2 & 144 & $80,384.86$ & $80,385 \pm 15$ & $80,363 \pm 20$ \\
$\mathrm{Z}^{0}$ & 12 & 12 & -7 & 144 & $91,187.56$ & $91,187.6 \pm 2.1$ & $91,187.4 \pm 2.1$ \\
$\mathrm{H}^{0}$ & 12 & 12 & -19 & 144 & $125,206.55$ & $125,500 \pm 400 \pm 400$ & None \\
\hline
\end{tabular}

The results strongly agree with measurement data, in particular for the weak force bosons; but interestingly the computed mass of the $\mathrm{W}^{ \pm}$is out of the SM prediction.

The electron mass is known with a relative precision of $2.210^{-8}$; it is the largest source of error in our calculus and the impact is proportional. It also impacts $D_{W Z}$ and $D_{H}$ but with lesser effects. Hence, assuming the $\mu$ of equation (1) is negligible for such heavy particles, we get a precision better than $10^{-7}$.

\subsection{Quarks Masses}

The quark masses are computed in Table 2 but the coefficients only come from phenomenology, and it would not be honest to mention it as a prediction without more arguments. But now the argument is coherence, because we have a complete picture in which there is no free parameter that can be adjusted. The relative precision should also be better than $10^{-7}$ and depend on the precision of the electron mass measurement. But we do not know the quark equivalent of the electron's $\mu$ at all. Its relative impact should logically be small on the heavy quarks, but larger and then probably not negligible on the light ones.

\subsection{Probably Nothing Else}

Our analysis gives some arguments against a fourth generation. The ratio between $1 / N P$ and $K D$ is related to geometry, and it should be impossible to have a correction larger than the resonance length; we should even reasonably expect $|K D|<<1 / N P$ (or $|N P K D|<<1$ ). But in practice we find large values; they are:

$$
\begin{gathered}
\text { Tau: } N P K D=0.346 \\
\text { Top: } N P K D=-0.588 \\
\text { Higgs: } N P K D=-0.153
\end{gathered}
$$

Leptons: We have $K>0$; when $K D$ exceeds the resonance length the resonance cannot happen because it would correspond to a sub-harmonic. As seen section 4, the next leptons generation should use $N=P=17$ and $K=7$, but then it gives $N P K D>1$ by far; hence no fourth lepton generation. 
Quarks: The only prime divisors of 266 are 2, 7, and 19; hence considering table 2, the only remaining divisor of 266 is 133; using (1) and $K=-6$ gives a negative volume $(|N P K D|>1)$; hence no fourth quark generation.

Bosons: We showed indirectly from (6-7) that $N P=144$ is necessarily unique. The obvious approach to guessing higher bosons masses is then to repeat the unused quarks $N s$ into the bosons $K s$. But the mass depends on their inner circulation which is unknown; then using $(14-15)$, we get two possible masses for each. They are:

$$
\begin{array}{lllll}
K & =-19 / 7 \rightarrow 81.494 & \text { or } & 81.820 & \mathrm{GeV} / \mathrm{c}^{2} \\
K & =-14 \rightarrow 109.012 & \text { or } & 109.821 & \mathrm{GeV} / \mathrm{c}^{2} \\
K=-38 \rightarrow 226.704 & \text { or } & 230.730 & \mathrm{GeV} / \mathrm{c}^{2}
\end{array}
$$

The masses predicted in (17) are in domains that have been excluded - hence no additional massive boson should be expected. Considering other divisors of 266, we find that taking $K=-133$ gives $|N P K D|>1$.

Other unknown particles could exist (massive or not) with remaining combinations of elementary currents.

Unknown massive particles: Three possibilities remain not breaking our rules; all correspond to fractional charges with 2 or 3 time-currents; they are: $+e / 3:\left[\uparrow^{+} \downarrow_{+}\right]$and $\left[\downarrow_{-} \uparrow^{+} \uparrow^{-}\right] ;+2 e / 3:\left[\uparrow^{+} \downarrow_{+} \downarrow_{-}\right]$and similar negative charges. The former $\left[\uparrow^{+} \downarrow_{+}\right]$is seen as a Cramer wave. Others should be quarks as seen in section 4 .

Unknown massless particles: Only four combinations remain: $[\lambda-\lambda+\measuredangle-]$ and the likes; those are eliminated in the SU(3) arithmetic where we take the virtual photons out, but they could also be something entirely new if they exist in a free state. There should be two current, one might be tachyonic (the scissor effect of [ $\nearrow-\swarrow-]$ ) while the physical currents are light speed (the arrows), including the $[\lambda+]$ which gives a true charge displacement.

Now the question is about the possible existence of other symmetries and modes of resonance. We find nothing else combining time-currents; all that remains in theory is the possibility of a resonance of the $X$-field alone.

\subsection{Manipulating the Field Below}

The existence of the field below is the main prediction in this paper. It is made of charged currents (space or time) which are sensitive to each other, and then to electricity. Then it can be manipulated with electricity; there is nothing new here because this is what particles do on the micro scale, as well as any material on a large scale.

But the field is also at the origin of inertia and of strength potentially equal to any known force. It means manipulating inertia and also directly the field energy (or the way it makes observable energy). In order to realize this, we need to play with symmetries; more exactly, we must play at tricking the game that makes them.

It seems impossible in the SM theoretical context essentially because it assumes fundamental symmetries, not emergences. But it becomes a natural matter of research if the SM symmetries emerge from a single field in which particles charges create some geometrical order.

If possible, it should be a question of geometry, not of brute force, and then a property of some materials. It is not difficult to find experimental results that seem to agree with this possibility; but as far as we know, none is reproduced at this time. Cautious filtering leads to consider a convergence of anomalies in a few experiments involving YBCO superconductors (which, by the way, are at the origin of our research and of equation (1)).

\section{The Nature of the Field, Lorentz Invariance}

We did not directly address the nature of the field below, in particular its charge quanta if any. It was done on purpose because the results and predictions only depend on coefficients, resonance numbers and geometry. It is discussed in this section; it can be seen as an interpretation - which we avoided as much as possible until now.

Up to now, we have neglected the fundamental resonance is given by the value of $X$ in equation (1); it corresponds to the resonance numbers $N=P=1$, probably $K=0$, and a self-energy $\mu / 2$ or null. It gives:

$$
m=X=8.145104 \mathrm{KeV} / \mathrm{c}^{2}
$$

The existence of such massive particle is quite unlikely, but even if purely theoretical it is fundamental and then it should be compared to $\mu$ which, in our model, comes from the self-interaction of the time-currents and then considering an electron from the product $e^{2}$. The rest of the electron mass $(N=P=K=2)$ is given by space currents and, with a unique field, it should corresponds to a product of charges, then in (18) the numbers $(N=P=$ 1) should correspond to a particle where a current $G$ is interacting with $e / 2$ which mass is a product $G e / 2$.

We then have the following correspondences:

$$
\begin{gathered}
G e / 2 \leftrightarrow m \\
e^{2} \leftrightarrow \mu
\end{gathered}
$$


We divide (19.1) by (19.2) and we find:

$$
G+e=68.4051247 e \approx e / 2 \alpha
$$

We recognize the Dirac condition (Dirac, 1931), and a minimal charge $G_{D}=e / 2 \alpha \approx 68.5 e$. The addition of a charge $e$ is then natural in (20) as it now appears as a magnetic time-current. The discrepancy $\left(-1.6610^{-3}\right)$ is acceptable considering that (18) represents a hypothetical particle. Then we write:

$$
G+e=G_{D}=e / 2 \alpha
$$

The plank constant appears (at last) in a form that gives the nature of the field. Moreover, equations $(20-21)$ are coherent with the toy model, and importantly the logic is not circular. Hence the vertical arrows represent magnetic time-current appearing as electric charges because of their orientation in space-time (and two currents $e / 2$ must be merged in $G$ in the charge quantization procedure). As far as we know, the symmetry of electromagnetism it implies is foreign to existing theories. Moreover, from (21) the origin of spin is obvious.

Interestingly, using $K=-1$ instead of 0 reduces the discrepancy to $+0.810^{-3}$ which is of opposite sign and twice closer to the Dirac condition, and it cancels with $K \approx-2 / 3$. It suggests that $\alpha_{S}$ in (2) is a coefficient of magnetic currents separation at short range. Then $D_{e}=\alpha \alpha_{S}$ comes from space-currents compressing time-currents; it depends on the currents ratio since we have $D_{e}=\alpha_{S}\left(e / 2 G_{D}\right)$. Then $D_{q}-D_{e}=\alpha^{2} \alpha_{S}=\alpha_{S}\left(e / 2 G_{D}\right)^{2}$ corresponds to magnetic currents compressing a magnetic charge; the force and the quantized distances are in reverse proportions. The factor 2 relates to the time-current $e c / 2$, we find $C=X$, as deduced section 3; color charge is magnetic.

Now this is the electromagnetic field, and then it is Lorentz invariant. But all space currents are charged and light-speed; then we should find a charge transformation that gives the $m \rightarrow m \gamma$ of special relativity. Hence we can show how and why equation (1) is Lorentz invariant.

The quantum of current $g V=e c / 2$ comes from the Bohr model under the assumption that the electron mass and wave are magnetic currents (Consiglio , 2013). It is expressed in standard electromagnetic notations where the $\gamma$ is interpreted as time dilation and then removed. Now we must write the true equation:

$$
g V \gamma_{S}=e c / 2
$$

where since $V$ is the de Broglie wave phase velocity, we have $V=c^{2} / v>c$, and we use $\gamma_{S}=1 / \mathrm{N}\left(V^{2} / c^{2}-1\right)$ which is a tachyon's $\gamma$. We have $V \gamma_{S}=\gamma c$, and then (22) reads:

$$
g=e / 2 \gamma
$$

This is the point of view of a fixed observer seeing the particle resonance as a clock subject to time dilation, and then time dilation is an effect of the space currents transformation. It gives in absorption and emission:

$$
X \rightarrow X / \gamma
$$

Then we can read the de Broglie relation using a total charge $G_{D}$ which, by symmetry, transforms like $g$ in (23):

$$
\begin{gathered}
E=h v=4 \pi \text { e } G_{D} v / c \\
v_{0} \rightarrow v_{0} / \gamma \quad \text { (time dilation) } \\
v_{0} \rightarrow v_{0} \gamma \quad \text { (observable frequency) }
\end{gathered}
$$

Since $e G_{D} / c=h / 2=$ constant, time dilation (25.2) comes from the transformation of space currents which induce a resonance frequency variation. This set of equations is coherent with the de Broglie phase harmony theorem (de Broglie, 1924) which proves using special relativity that a stationary wave of frequency $v_{0}$ obeying (25.3) is always in phase with the particle clock (in de Broglie's words) at the particle location and obeying (25.2). On this basis we must identify the resonance with de Broglie's particle clock.

But we used the back door to introduce the $\gamma$ in equation (22); although this demonstration in not sufficient it gives a first idea of what is happening. But we understand now that the charge $g$ in the expression $g V=e c / 2$ is simply the projection on the electron's relativistic present of half of the sum of its time-currents $e c / 2$. When the electron velocity increases the time-currents become space-currents; then we use the Dirac condition and we write:

$$
G e / \sin (2 \theta)=h c / 2
$$

where $\theta$ is the angle between the electron time-currents and the light-cone on which the current $G$ is. But the introduction of $\sin (2 \theta)$ in this equation, although it is equivalent to the $1 / \gamma$, comes from the intersection of the space extension of the time-currents with the light-cone, not from a projection as it would be in Minkowski space.

Now since $h c / 2$ is a natural constant it gives $(25.2-25.3)$ in the form $e \rightarrow e, G \rightarrow G \gamma$ and also (25.1) in the opposite form $e \rightarrow e \gamma, G \rightarrow G$; it agrees with de Broglie. 
Now it is trivial to show that a stationary wave is given by the reflection of light-speed currents. Consider a random current $\varphi(r, t)$ approaching an electron, then delayed in its interaction of $\tau=\gamma / v_{0}=\tau_{0} \gamma$, in agreement with (25.2), and sent back in space and time at light speed. The phase of the current is:

$$
\varphi^{\prime}(r, t)=\varphi(r, t)+\varphi\left(r, t-\tau_{0} \gamma\right)
$$

The same reasoning applies for a current coming from the future. It shows how the particle imprints its Compton wavelength in the form of a modulation of an incoming current. But this equation agrees with time dilation (25.2); then using the phase harmony theorem, a fixed observer will see:

$$
\varphi^{\prime}(r, t)=\varphi(r, t)+\varphi\left(r, t-\tau_{0} / \gamma\right)
$$

But now the model depicts a new kind of space-time where time dilation is the effect on particles resonances of a transformation which only applies to light speed space currents. Technically, this is not Minkowski space, the concept is weaker and more general, but special relativity is proven by $(24-25)$ in a form compatible with the original Einstein theory (1905).

For time-currents, time dilation does not apply and $g V=e c / 2$ gives the up and down velocities "thru time":

$$
V_{U p}=4 \mathrm{c} / 3 ; V_{\text {Down }}=2 \mathrm{c} / 3
$$

The addition of which gives:

$$
V_{U p}+V_{\text {Down }}=2 c
$$

This is what we expect for a fixed observer seeing light speed currents coming from opposite directions. It shows how the transformation of a quantum of current gives the toy model assuming a breach in the time direction.

Finally we can discuss equation (3) - but we do not know what this coincidence is about. At first sight it should relate to energies, but the coefficients $4 \pi$ and $\sqrt{ } 2$ are not understood.

The electron mass must come from a product that we write:

$$
(G+i e / 2)(G-i e / 2)=G^{2}+e^{2} / 4 \rightarrow m_{e}
$$

where $e / 2$ are the currents, not the apparent charges; we use imaginary numbers and then the cross-products cancel. Then we identify the squared charges with the masses in (3); it gives:

$$
4 \pi \alpha^{2} G^{2}=\sqrt{ } 2 e^{2} / 4
$$

Assume $G=G_{D}$, we get:

$$
1=\sqrt{ } 2 / 4 \pi
$$

which is ridiculous and then the coincidence (3) does not relate to energy; but then multiplying each side by the Plank constant, this expression can be read:

$$
h \leftrightarrow \sqrt{ } 2 h / 2
$$

which, since the cross-products cancel in (29.1), we interpret as:

\section{One quantum of action $h$ at each period of an electron pulsation makes its spin}

The coincidence (3), when interpreted in this manner does not relate to masses; it links the electron spin to a true physical action. With respect to the resonance concept, with real physical resonances of space and time currents the existence of this equivalence is mandatory. Now in $(18-20-21)$ we used $e^{2}$ instead of $e^{2} / 4$, then equation (21) reads $4 G+e=G_{D}=e / 2 \alpha$; it does not change the significance of the coincidence.

\section{Answering Questions from the Referees}

Some questions were out of the initial scope of the paper. Answers are below.

Question 1: The observation of neutrino oscillations indicates that the neutrino has a non-zero mass (i.e. Data Bay experiments). What is the prediction of the present formalism?

Direct measurements of the neutrino velocity (from supernovae) are consistent with a null mass; the neutrino mass is a theoretical prediction which finally comes from interpretation in the standard theory. Measurements on earth show a continuous oscillation and then it implies statistical predictability, but they are all made in the presence of matter (air as a minimum); so they can be interpreted as matter "switching" the neutrino states - or neutrinos state varying "alone"; the question is currently open and some new physics is expected.

The question actually states that neutrino oscillation requires mass - hence it includes the assumption that something light-speed cannot evolve. It is a natural consequence of the standard theory; but in parallel it does not question why or how an entangled photon state is undetermined while it cannot change. 
The toy model shows a difference with photons since neutrinos have no response wave. In Cramer's interpretation, a photon is linked to its source, and this is the way to explain photons entanglement. The model predicts that the state of a neutrino is not constrained by the state of its source in the same manner as a photon. Hence its initial state is just given and can evolve between emission and absorption, whatever the reason, even if its velocity is light-speed; it predicts light-speed massless neutrinos which oscillations are not forbidden.

In section 6 it comes naturally to question the reality of Minkowski space. But instead of choosing between special relativity and neutrinos mass the theory is based on a weaker concept in which it is naturally included from the very beginning: In the reasoning to equation (1) the self-energy $\mu$ is assumed massless and light-speed (it is assumed later to be a product $e^{2} / 4$, and energy comes from a product and the current $e c / 2$ is really assumed massless).

Question 2: Causality.

Three aspects must be considered:

1) In this model, the time-currents break the symmetry of time, but the Cramer interpretation is "just" an interpretation of standard formalism and it does not enable causality violation.

2) The scissor effect of $[\nearrow-\swarrow-]$ and $[\nearrow+\swarrow+]$ is faster than light, but it does not break causality as long as it is the de Broglie wave of its source, including the wave direction (Consiglio , 2013). It is not the so called reinterpretation principle; it is just the Lorentz transformation of de Broglie's $V=c^{2} / v$. A particle can permanently throw free tachyons at this velocity and no causality violation will ever occur. This is a unique property and no other velocity is permitted. A rapid literature review shows that all mind experiments on causality violation assume that tachyons are thrown at any velocity in any direction.

3) The last aspect is space-currents going back in time; those are responses (reflections), then no information can be transferred and no energy can be sent back in time.

Question 3: The expression (1) is a resonance equation, the particle mass corresponds to the pole of the resonance. What about the width of the resonances? Are widths in agreement with particles lifetime?

Massive Bosons: In order to compute the bosons widths, we need to understand the mechanical limits at which the resonance breaks. The $\mathrm{W}^{ \pm}$and $\mathrm{Z}^{0}$ are easy cases as their mass depends on $K$ only, the product $N P$ is constant and the geometry is understood (section 2, equations 7.x); firstly change the resonance with $K \rightarrow(K+1+1 / 24)$, this is the energy at which the resonance breaks because:

- The $(K+1)$ corresponds to a difference 1 in the $(266-K)$ of equations (7.x).

- The $1 / 24$ is half of $1 / 12$ and it is natural; we cannot take the full $1 / 12$ since this is the retroaction: $1 / 12$ is equivalent to a full period of a sine potential centered on 0 ; hence $1 / 24$.

Now we compute the masses of those faked resonances and take the difference with the computed masses of the real one; this is the width in which the resonance holds. It gives:

- $\quad \mathrm{W}^{ \pm}, K=-2 \rightarrow K=(-2+1+1 / 24) \rightarrow 2.085 \mathrm{GeV}$ (a perfect match with existing data).

- $\quad Z^{0}, K=-7 \rightarrow K=(-7+1+1 / 24) \rightarrow 2.468 \mathrm{GeV}$ ( $1 \%$ less than the existing data).

The Higgs has four currents spinning in two opposite directions and the picture is quite different. With respect to the $\mathrm{W}^{ \pm}$and the $\mathrm{Z}^{0}$ where two currents have the same rotation (spin 1 ), four currents and two opposite rotations (spin 0 ) imply firstly to remove the " +1 " and secondly to use $1 / 144$ instead of $1 / 12$. Now as found in (9) the retroaction coefficient is $1 / 6$. The width coefficient is then given by $1 /(144 \times 6)$, and then:

- $\quad \mathrm{H}^{0}, K=-19 \rightarrow K=(-19+1 /(144 \times 6)) \rightarrow 4.11 \mathrm{MeV}(2.5 \%$ less than the SM prediction).

The SM predicts is $4.21 \mathrm{MeV}$ but at $126 \mathrm{GeV}$ - so the difference is actually lesser.

Top Quark: Quarks are sub-harmonics of a high resonance $N=266$ on the circular path, the path is reduced by the $K=-6$. This is the physical constraint and then also the resonances to break; it suggests $(K+1 / 266)$ and the same problem as for bosons: the width comes from the circular path. The radial resonance corresponding to $P=3$ is on the radial path, and then unbreakable. But it requires a phase lock with the circular one; it increases the coefficient proportionally to $P=3$ thus reducing stability; the coefficient is now $(K+3 / 266)$. The resonance is mixed with two orthogonal paths. The path length is then multiplied by $\sqrt{ } 2=|1+i|$; hence the width is:

$$
\text { Top: } K=(-6+3 \sqrt{2} / 266) \rightarrow 1.97 \mathrm{GeV} \text {; compare with experimental data: } 2.0 \pm 0.5 \mathrm{GeV} \text {. }
$$

The result is appropriate and the logic is in agreement with a mixed resonance.

Leptons: The double radial resonance of leptons should be locally unbreakable; they address directly the mechanism of query/response. Computing their stability should not be a problem of resonance numbers. 


\section{Conclusions}

In the spirit of the standard theory, the fact that the elementary particles mass spectrum can be computed (or so well approximated) using equation (1) is an anomaly or a suite of coincidences - twelve of them. In this paper, we show that the resonance numbers are coherent with geometry and that all the coefficients are coherent with each other. The theory gives an almost classical origin to all mass ratios and to the bosons and the top quark widths. It is physically and quantitatively coherent, it is minimal, it cannot be tuned, it is predictive, and then it cannot be a suite of random coincidences. This is new physics - as always, it comes from unexplained phenomenology!

Hence the main interest of this paper is in the very existence of the field below, firstly because it could lead to applications that cannot be imagined in the SM theoretical context - but not only. The Feynman-Wheeler absorber theory considers exchanges of energy, and the equation (1) was initially found considering quantized momentum exchanges (Consiglio , 2012), so called Universons (Poher \& Marquet, 2012) which is close to the same idea.

But in both cases, it requires very unnatural solutions when it comes to inertia, Lorentz invariance, and conservation laws. It leads to considering charged currents but then it questions the very nature of energy and momentum - a question which is definitely out of the scope of the standard theory and of its extensions, because those are merely conserved mathematical quantities with no invariant physical form. In other words, we hunt the quantum ghost and we begin to see its face. It is out of reach of quantum theories because those are, since the very beginning and the so called Copenhagen interpretation, mathematical abstractions designed on and possibly because of our ignorance of its nature. It makes the standard theory an emergent description of an underlying reality; it is predictive because the Lagrangians are the natural connections with the field below, but the formalism cannot provide with clear insight to its nature.

Finally this paper is not about particles mass or a classical unification of the SM forces but on the interpretation of the known fields. All are found to be the same field taking different forms - so called resonances. It means that in order to extend the standard theory we must look in a new direction.

\subsection{Addendum - On Methodology}

In results, methodology, and directions, Dirac was one of the most influential physicists of the $20^{\text {th }}$ century; he wrote (Dirac, 1931, section 1):

"There are at present fundamental problems in theoretical physics awaiting solution; (...) the solution of which problems will presumably require a more drastic revision of our fundamental concepts than any that have gone before. Quite likely these changes will be so great that it will be beyond the power of human intelligence to get the necessary new ideas by direct attempts to formulate the experimental data in mathematical terms.

We can first easily guess that Dirac (and some of his successors) did not have all the data - far from it. Then:

"The theoretical worker in the future will therefore have to proceed in a more indirect way. The most powerful method of advance that can be suggested at present is to employ all the resources of pure mathematics in attempts to perfect and generalize the mathematical formalism that forms the existing basis of theoretical physics, and after each success in this direction, to try to interpret the new mathematical features in terms of physical entities."

The solution we propose comes exactly at the opposite of Dirac's suggestion ans in full agreement with the method that he could not use: We use physical reasoning to directly formulate the experimental data in mathematical form; and then we extract more detailed laws from the results and the parameters. At this point powerful mathematical abstractions become possible and useful.

Hence we conjecture: A physical action is a product of currents of the field below. Conservation laws imply alternative algebras.

According to the Hurwitz theorem (Dickson, 1919) there exits four division algebra consisting of R (real numbers), $\mathrm{C}$ (complex numbers), $\mathrm{H}$ (quaternions) and $\mathrm{O}$ (octonions). Then R corresponds to energy, $\mathrm{C}$ to $\mathrm{U}(1)$, $\mathrm{H}$ to $\mathrm{SU}(2)$ and $\mathrm{O}$ to $\mathrm{SU}(3)$. According to the conjecture above, those are the only pure symmetries in nature.

For instance, let us write (29.1) in quaternion form:

$$
-j((G+i e / 2)(j G-k e / 2))=\left(G^{2}+e^{2} / 4\right)-(i+1) e G / 2 \rightarrow m_{e}+\operatorname{spin}
$$

This form is appropriate since according to (21), each time-current of an electron sees the other on the other side of the light-speed singularity - currents are rotated to an imaginary plane, hence quaternions. The spin comes with a norm $\sqrt{ } 2$; the observable real part is $h / 2$ and then $e G=\hbar c$. It agrees directly with our reasoning on equation (3). 


\section{References}

ATLAS Collaboration. (2013). Combined measurements of the mass and signal strength of the Higgs-like boson with the ATLAS detector using up to $25 \mathrm{fb}-1$ of proton-proton collision data. Retrieved from http://cds.cern.ch/record/1523727

Consiglio, J. (2012). On particles mass and the Universons hypothesis. Applied Physics Research, 4(2), 144-158. http://dx.doi.org/10.5539/apr.v4n2p144

Consiglio, J. (2013). Minkowski space and Tachyons velocity. Applied Physics Research, 5(6), 1-14. http://dx.doi.org/10.5539/apr.v5n6p1

Consiglio, J. (2014). Below the Standard Model? Applied Physics Research, 6(2), 19-27. http://dx.doi.org/10.5539/apr.v6n2p19

Cramer, J. (1986). The transactional interpretation of quantum mechanics. Rev. Mod. Phys., 58(3). http://link.aps.org/doi/10.1103/RevModPhys.58.647. DOI: 10.1103/RevModPhys.58.647

De Broglie, L. (1924). Recherches sur la théorie des quanta. Annales de Physique - 10e sérié - Tome III Janvier-Février 1925.

Dickson, L.E, (1919) "On Quaternions and Their Generalization and the History of the Eight Square Theorem", Ann. Math., 20, (1919), 155.

Dirac, P. A. M. (1931). Quantized singularities in the Electromagnetic Field, Proc. Roy. Soc., A(133), 60.

Einstein, A. (1905). Zur Elektrodynamik bewegter Körper. Annalen der Physik, 1905.

Mohr, P. J. (2012). CODATA Recommended Values of the Fundamental Physical Constants: 2010. Retrieved from http://arxiv.org/pdf/1203.5425.pdf

Poher, C., \& Marquet, P. (2012). General Relativity and Universons. Applied Physics Research, 4(1), 120. http://dx.doi.org/10.5539/apr.v4n1p120

Poincaré, H. (1906). Sur la dynamique de l'électron. Rendiconti del Circolo Matematico di Palermo, 21(1906), 129-176.

The ATLAS, CDF, CMS and D0 Collaborations. (2013). First combination of Tevatron and LHC measurements of the top-quark mass. arXiv, 1403, 4427

The CMS Collaboration. (2014). Measurement of the top-quark mass in top-quark pair events with lepton+jets final states in pp collisions at sqrt(s) $=8$ TeV. CMS Physics Analysis Summary TOP-14-001. Retrieved from https://cds.cern.ch/record/1690093? ln=en

\section{Copyrights}

Copyright for this article is retained by the author(s), with first publication rights granted to the journal.

This is an open-access article distributed under the terms and conditions of the Creative Commons Attribution license (http://creativecommons.org/licenses/by/3.0/). 